\title{
Governing equations of transient soil water flow and soil water flux in multi-dimensional fractional anisotropic media and fractional time
}

\author{
M. Levent Kavvas, Ali Ercan, and James Polsinelli \\ Hydrologic Research Laboratory, Department of Civil \& Environmental Engineering, University of California, \\ Davis, CA 95616, USA \\ Correspondence to: M. Levent Kavvas (mlkavvas@ucdavis.edu)
}

Received: 2 September 2016 - Discussion started: 26 September 2016

Revised: 27 January 2017 - Accepted: 22 February 2017 - Published: 13 March 2017

\begin{abstract}
In this study dimensionally consistent governing equations of continuity and motion for transient soil water flow and soil water flux in fractional time and in fractional multiple space dimensions in anisotropic media are developed. Due to the anisotropy in the hydraulic conductivities of natural soils, the soil medium within which the soil water flow occurs is essentially anisotropic. Accordingly, in this study the fractional dimensions in two horizontal and one vertical directions are considered to be different, resulting in multi-fractional multi-dimensional soil space within which the flow takes place. Toward the development of the fractional governing equations, first a dimensionally consistent continuity equation for soil water flow in multidimensional fractional soil space and fractional time is developed. It is shown that the fractional soil water flow continuity equation approaches the conventional integer form of the continuity equation as the fractional derivative powers approach integer values. For the motion equation of soil water flow, or the equation of water flux within the soil matrix in multi-dimensional fractional soil space and fractional time, a dimensionally consistent equation is also developed. Again, it is shown that this fractional water flux equation approaches the conventional Darcy equation as the fractional derivative powers approach integer values. From the combination of the fractional continuity and motion equations, the governing equation of transient soil water flow in multidimensional fractional soil space and fractional time is obtained. It is shown that this equation approaches the conventional Richards equation as the fractional derivative powers approach integer values. Then by the introduction of the Brooks-Corey constitutive relationships for soil water into
\end{abstract}

the fractional transient soil water flow equation, an explicit form of the equation is obtained in multi-dimensional fractional soil space and fractional time. The governing fractional equation is then specialized to the case of only vertical soil water flow and of only horizontal soil water flow in fractional time-space. It is shown that the developed governing equations, in their fractional time but integer space forms, show behavior consistent with the previous experimental observations concerning the diffusive behavior of soil water flow.

\section{Introduction}

Various laboratory (Silliman and Simpson, 1987; Levy and Berkowitz, 2003) and field studies (Peaudecerf and Sauty, 1978; Sudicky et al., 1983; Sidle et al., 1998) of transport in subsurface porous media have shown significant deviations from Fickian behavior. As one approach to the modeling of the generally non-Fickian behavior of transport, Meerschaert, Benson, Baumer, Schumer, Zhang and their coworkers (Meerschaert et al., 1999, 2002, 2006; Benson et al., 2000a, b; Baumer et al., 2005, 2007; Schumer et al., 2001, 2009; Zhang et al., 2007, 2009; Zhang and Benson, 2008) have introduced the fractional advection-dispersion equation (fADE) as a model for transport in heterogeneous subsurface media. By theoretical and numerical studies the above authors have shown that fADE has a nonlocal structure that can model well the heavy-tailed non-Fickian dispersion in subsurface media, mainly by means of a fractional spatial derivative in the dispersion term of the equation. Mean- 
while, they have also shown that fADE, with a fractional time derivative, can also model well the long particle waiting times in transport in both surface and subsurface environments. However, while the above-mentioned studies provided extensive treatment of the fractional differential equation modeling of transport in fractional time-space by subsurface flows, few studies have addressed the detailed modeling of the actual subsurface flows in porous media in fractional time-space.

He (1998) seems to be the first scholar who proposed a fractional form of Darcy's equation for water flux in porous media. Based on this fractional water flux equation, in his pioneering work He (1998) then proposed a fractional governing equation of flow through saturated porous media. The left-hand side (LHS) and the right-hand side (RHS) of He's fractional Darcy flux formulation have different units. As saturated flow equations, He's proposed governing equations address the groundwater flow instead of the unsaturated soil water flow. Since the focus of our study is soil water flow in fractional time-space, below we shall discuss the literature that specifically addresses the fractional soil water flow equations.

As early as in 1960s Gardner and his co-workers (Ferguson and Gardner, 1963; Rawlins and Gardner, 1963) questioned the classical diffusivity expression in the diffusion form of the conventional Richards equation for soil water flow being only dependent on the soil water content. Based on their experimental observations, they reported that diffusivity was also dependent explicitly on time besides being dependent on the soil water content. Following on these experimental observations, Guerrini and Swartzendruber (1992) hypothesized a new form for Richards equation for horizontal unsaturated soil water flow in semi-rigid soils. Unlike the assumption that the soil hydraulic conductivity $K$ and soil water pressure head $\psi$ are only dependent on the soil water content, they hypothesized that $K$ and $\psi$ are also dependent explicitly on time. This hypothesis led them to the formulation of the diffusivity coefficient $D$ within the diffusion form of the Richards equation as function of not only the soil water content but also explicitly on time, that is $D=D(\theta, t)=$ $E(\theta) t^{m}$, where $E$ is a function of water content $\theta$ while $m$ is a power value. The application of their theory to the field data of Rawlins and Gardner (1963) proved successful, yielding fractional values of $m$ less than unity in $t^{m}$. In a field experimental study of horizontal water absorption into porous construction materials (fired-clay and siliceous brick), El-Abd and Milczarek (2004) arrived at a formulation of diffusivity coefficient again in the form $D(\theta, t)=E(\theta) t^{m}$. The application of this form to their experimental data produced satisfactory results.

The study by Pachepsky et al. (2003) appears to be the first to propose a fractional model of horizontal, unsaturated soil water flow in field soils. Motivated by the observations of Nielsen et al. (1962) on the jerky movements of the infiltration front in field soils, which can be explained by long recurrence time intervals in between motions, Pachepsky et al. (2003) proposed a time-fractional model of horizontal soil water flow in field soils. While the space component of their model has integer derivatives, they proposed a fractional form for the diffusivity, and expressed the Darcy water flux formulation in diffusive form with their proposed fractional diffusivity. Pachepsky et al. (2003) showed that the cause for fractional diffusivity is the scaling of time in the Boltzmann relationship not with the power of 0.5 (which corresponds to Brownian motion) but with a power less than 0.5 , an experimental observation that was already made by Guerrini and Swartzendruber (1992). Pachepsky et al. (2003) supported their claim by various previous experimental studies' results, and showed that their proposed time-fractional form of the Richards equation with fractional diffusivity can explain experimental data. Meanwhile, Gerolymatou et al. (2006) proposed a fractional integral form for the Richards equation in fractional time but in integer horizontal space for unsaturated soil water flow in one horizontal dimension. Comparing their model simulations against the field experimental data of ElAbd and Milczarek (2004), they showed that their fractional Richards equation describes the evolution of soil water content in time and space better than the corresponding integer Richards equation. Again considering horizontal unsaturated soil water flow in fractional time but integer space, Sun et al. (2013) utilized the concept of fractal ruler in time, due to Cushman et al. (2009), to define a fractional derivative in time which they used to modify the integer time derivative in the conventional Richards equation. By means of this fractional derivative definition they were able to model the anomalous Boltzmann scaling in the wetting front movement and were able to obtain good fits to water content experimental data. Sun et al. (2013) conjectured that the time-dependent diffusivity $D(\theta, t)=E(\theta) t^{m}$ (for a fractional value of $m$ ) due to Guerrini and Swartzendruber (1992) and El-Abd and Milczarek (2004), in the conventional Richards equation can be expressed essentially by representing the conventional integer derivative of the soil water content with respect to time by a product of the fractional time derivative of the soil water content and a fractional power of time.

The above-cited studies on the governing equations of soil water flow only treat time with fractional dimension, while keeping space with integer dimension. Furthermore, these studies address only one spatial dimension. Accordingly, our study in the following will attempt to develop a fractional continuity equation and a fractional water flux (motion) equation for unsaturated soil water flow in both fractional time and in multi-dimensional fractional space, starting from the conventional mass conservation and Darcy's law. Due to the anisotropy in the hydraulic conductivities of natural soils, the soil medium within which the soil water flow occurs is essentially anisotropic. Accordingly, in this study the fractional dimensions in two horizontal and one vertical directions will be considered different, resulting in multi-fractional space within which the flow takes place. To- 
ward the development of the fractional governing equations, first a dimensionally consistent continuity equation for soil water flow in multi-fractional, multi-dimensional space and fractional time will be developed. For the motion equation of soil water flow, or the equation of water flux within the soil matrix in multi-fractional multi-dimensional space and fractional time, a dimensionally consistent equation will also be developed. From the combination of the fractional continuity and motion equations, the governing equation of transient soil water flow in multi-fractional, multi-dimensional space and fractional time will be obtained. It will be shown that this equation approaches the conventional Richards equation as the fractional derivative powers approach integer values. Then by the introduction of the Brooks-Corey constitutive relationships for soil water (Brooks and Corey, 1964) into the fractional transient soil water flow equation, an explicit form of the equation will be obtained in multi-dimensional, multifractional space and fractional time. The governing fractional equation is then specialized to the case of only vertical soil water flow and of only horizontal soil water flow in fractional time-space.

\section{Derivation of the continuity equation for transient soil water flow in multi-dimensional fractional space and fractional time}

Let $D_{a}^{k \beta} f(x)$ be a Caputo fractional derivative of the function $f(x)$, defined as (Podlubny, 1999; Odibat and Shawagfeh, 2007; Usero, 2008; Li et al., 2009)

$$
\begin{aligned}
D_{a}^{k \beta} f(x) & =\frac{1}{\Gamma(m-k \beta)} \int_{a}^{x} \frac{f^{m}(\xi)}{(x-\xi)^{k \beta+1-m}} \mathrm{~d} \xi, \\
m-1<\beta & <m, m \in N, x \geq a .
\end{aligned}
$$

Specializing the integer $m=1$ reduces Eq. (1) to

$D_{a}^{k \beta} f(x)=\frac{1}{\Gamma(1-k \beta)} \int_{a}^{x} \frac{f^{\prime}(\xi)}{(x-\xi)^{k \beta}} \mathrm{d} \xi, 0<\beta<1, x \geq a$.

Then to $\beta$-order

$$
D_{a}^{\beta} f(x)=\frac{1}{\Gamma(1-\beta)} \int_{a}^{x} \frac{f^{\prime}(\xi)}{(x-\xi)^{\beta}} \mathrm{d} \xi, 0<\beta<1, x \geq a .
$$

One can obtain a $\beta$-order approximation to a function $f(\cdot)$ around " $a$ " as

$$
f(x)=f(a)+\frac{(x-a)^{\beta}}{\Gamma(\beta+1)} D_{a}^{\beta} f(x), 0<\beta<1 .
$$

This result follows by taking the upper limit value of the Caputo derivative at " $x$ " in the mean value representation of a function in terms of fractional Caputo derivative (Usero, 2008; Li et al., 2009; Odibat and Shawagfeh, 2007) in order to have a distinct value for the above $\beta$-order approximation of the function $f$ around " $a$ ". Within this framework the governing equations, based on this approximation, become prognostic equations that shall be known from the outset of model simulation for the whole time-space modeling domain. The next issue is what to take for the value of " $a$ ". If one expresses Eq. (4) with $a=x-\Delta x$, that is,

$f(x)=f(x-\Delta x)+\frac{(\Delta x)^{\beta}}{\Gamma(\beta+1)} D_{x-\Delta x}^{\beta} f(x)$,

then the evaluation of the Caputo fractional derivative for $f(x)=x$ will result in an expression that will contain a binomial expansion with a fractional power, which has infinite number of terms. As will be discussed in a later section, in order to render the developed fractional governing equations to become purely differential equations, it is necessary to establish an analytical relationship between $\Delta x$ and $(\Delta x)^{\beta}$ that will be universally applicable throughout the modeling domain. This is possible when one takes the lower limit in the above Caputo derivative in Eq. (5) as zero (0) (that is, $\Delta x=x)$ for $f(x)=x$. Then under such a construct, it will be possible to develop purely differential forms (with only fractional differential operators and no finite difference operators) for the governing equations of soil water flow, as will be shown in the following.

Within the above framework one can express the net mass outflow rate from the control volume in Fig. 1 as

$\left[\rho q_{x_{1}}\left(x_{1}, x_{2}, x_{3} ; t\right)-\rho q_{x_{1}}\left(x_{1}-\Delta x_{1}, x_{2}, x_{3} ; t\right)\right] \Delta x_{2} \Delta x_{3}$

$+\left[\rho q_{x_{2}}\left(x_{1}, x_{2}, x_{3} ; t\right)-\rho q_{x_{2}}\left(x_{1}, x_{2}-\Delta x_{2}, x_{3} ; t\right)\right] \Delta x_{1} \Delta x_{3}$

$+\left[\rho q_{x_{3}}\left(x_{1}, x_{2}, x_{3} ; t\right)-\rho q_{x_{3}}\left(x_{1}, x_{2}, x_{3}-\Delta x_{3} ; t\right)\right] \Delta x_{1} \Delta x_{2}$.

Then by introducing Eq. (5) into Eq. (6) with $\Delta x=x$, and expressing the resulting Caputo derivative $D_{0}^{\beta} f(x)$ (taking $\Delta x=x$ renders the lower limit in the Caputo derivative of Eq. 5 to be 0$)$ by $\frac{\partial^{\beta} f(x)}{(\partial x)^{\beta}}$ for convenience, the net mass flux from the soil control volume in Fig. 1 may be expressed to $\beta$-order in fractional space as

$$
\begin{aligned}
& =\frac{\left(\Delta x_{1}\right)^{\beta_{1}}}{\Gamma\left(\beta_{1}+1\right)}\left(\frac{\partial}{\partial x_{1}}\right)^{\beta_{1}}\left(\rho q_{x_{1}}\left(x_{1}, x_{2}, x_{3} ; t\right)\right) \Delta x_{2} \Delta x_{3} \\
& +\frac{\left(\Delta x_{2}\right)^{\beta_{2}}}{\Gamma\left(\beta_{2}+1\right)}\left(\frac{\partial}{\partial x_{2}}\right)^{\beta_{2}}\left(\rho q_{x_{2}}\left(x_{1}, x_{2}, x_{3} ; t\right)\right) \Delta x_{1} \Delta x_{3} \\
& +\frac{\left(\Delta x_{3}\right)^{\beta_{3}}}{\Gamma\left(\beta_{3}+1\right)}\left(\frac{\partial}{\partial x_{3}}\right)^{\beta_{3}}\left(\rho q_{x_{3}}\left(x_{1}, x_{2}, x_{3} ; t\right)\right) \Delta x_{1} \Delta x_{2},
\end{aligned}
$$

where different fractional powers are considered in the three Cartesian directions in space due to the general anisotropy in the soil permeabilities and in the resulting flows in the soil media. It also follows from Eq. (5) with $f\left(x_{i}\right)=x_{i}$ that to $\beta$-order one obtains the approximation

$\Delta x_{i}=\frac{\left(\Delta x_{i}\right)^{\beta_{i}}}{\Gamma\left(\beta_{i}+1\right)} \frac{\partial^{\beta_{i}} x_{i}}{\left(\partial x_{i}\right)^{\beta_{i}}}, \quad i=1,2,3$.

With respect to the Caputo derivative $D_{0}^{\beta} x$,

$$
\frac{\partial^{\beta_{i}} x_{i}}{\left(\partial x_{i}\right)^{\beta_{i}}}=\frac{x_{i}^{1-\beta_{i}}}{\Gamma\left(2-\beta_{i}\right)}, \quad i=1,2,3 .
$$




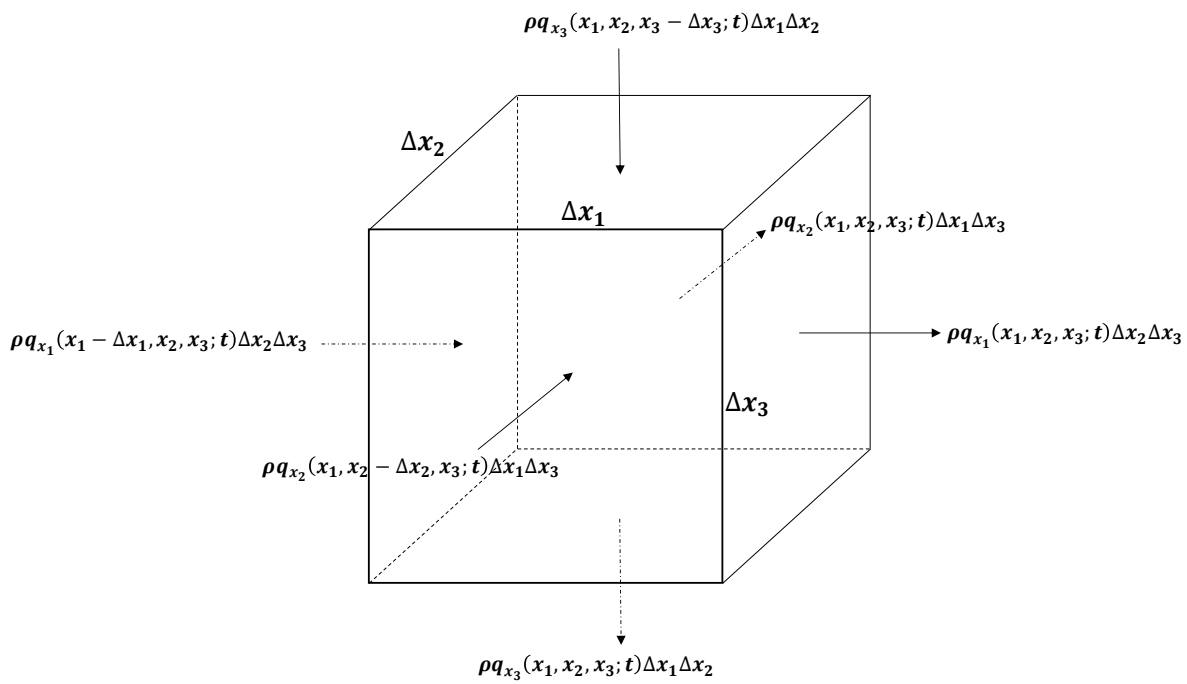

Figure 1. The control volume for the three-dimensional soil water flow.

Hence, combining Eqs. (8) and (9) yields

$$
\left(\Delta x_{i}\right)^{\beta_{i}}=\frac{\Gamma\left(\beta_{i}+1\right) \Gamma\left(2-\beta_{i}\right)}{x_{i}^{1-\beta_{i}}}\left(\Delta x_{i}\right), \quad i=1,2,3
$$

with respect to $\beta_{i}$-order fractional space in the $i$ th direction, $i=1,2,3$.

Introducing Eq. (10) into Eq. (7) yields for the net mass outflow rate

$$
\begin{aligned}
& =\frac{\Gamma\left(2-\beta_{1}\right)}{x_{1}^{1-\beta_{1}}}\left(\frac{\partial}{\partial x_{1}}\right)^{\beta_{1}}\left(\rho q_{x_{1}}(\bar{x} ; t)\right) \Delta x_{1} \Delta x_{2} \Delta x_{3} \\
& +\frac{\Gamma\left(2-\beta_{2}\right)}{x_{2}^{1-\beta_{2}}}\left(\frac{\partial}{\partial x_{2}}\right)^{\beta_{2}}\left(\rho q_{x_{2}}(\bar{x} ; t)\right) \Delta x_{1} \Delta x_{2} \Delta x_{3} \\
& +\frac{\Gamma\left(2-\beta_{3}\right)}{x_{3}^{1-\beta_{3}}}\left(\frac{\partial}{\partial x_{3}}\right)^{\beta_{3}}\left(\rho q_{x_{3}}(\bar{x} ; t)\right) \Delta x_{1} \Delta x_{2} \Delta x_{3}, \\
& \bar{x}=\left(x_{1}, x_{2}, x_{3}\right)
\end{aligned}
$$

to $\beta$-order, reflecting multi-fractional scaling in the anisotropic soil medium.

Denoting the volumetric water content by $\theta(\bar{x}, t)$, the water volume $V_{\mathrm{w}}$ within the control volume in Fig. 1 may be expressed as

$V_{\mathrm{w}}=\theta \Delta x_{1} \Delta x_{2} \Delta x_{3}$.

Hence, the time rate of change of mass within the control volume in Fig. 1 is

$$
\frac{\rho(\bar{x}, t) \theta(\bar{x}, t)-\rho(\bar{x}, t-\Delta t) \theta(\bar{x}, t-\Delta t)}{\Delta t} \Delta x_{1} \Delta x_{2} \Delta x_{3} .
$$

Introducing Eq. (5) with fractional power $\beta$ replaced by $\alpha$, $x$ replaced by $t$ and with $\Delta t=t$, into Eq. (13), and expressing the resulting Caputo derivative operator with its lower limit as 0 , by $\frac{\partial^{\alpha}}{(\partial t)^{\alpha}}$ for convenience, yields the time rate of change of mass within the control volume with respect to $\alpha$ fractional time increments

$\frac{(\Delta t)^{\alpha}}{\Delta t \Gamma(\alpha+1)}\left(\frac{\partial}{\partial t}\right)^{\alpha} \rho(\bar{x}, t) \theta(\bar{x}, t)$

to $\alpha$-order. With respect to the Caputo derivative $D_{0}^{\alpha} t=$ $\frac{\partial^{\alpha} t}{(\partial t)^{\alpha}}$

$\frac{\partial^{\alpha} t}{(\partial t)^{\alpha}}=\frac{t^{1-\alpha}}{\Gamma(2-\alpha)}$,

which when combined with Eq. (5) (with $x$ replaced by $t$ and $\beta$ replaced by $\alpha$ ) yields the approximation

$(\Delta t)^{\alpha}=\frac{\Gamma(\alpha+1) \Gamma(2-\alpha)}{t^{1-\alpha}}(\Delta t)$

to $\alpha$-order. Introducing Eq. (16) into Eq. (14) yields for the time rate of change of mass within the control volume in Fig. 1 with respect to $\alpha$-order fractional time increments:

$\frac{\Gamma(2-\alpha)}{t^{1-\alpha}} \frac{\partial^{\alpha} \rho(\bar{x}, t) \theta(\bar{x}, t)}{(\partial t)^{\alpha}} \Delta x_{1} \Delta x_{2} \Delta x_{3}$.

Since the time rate of change of mass within the control volume of Fig. 1 is inversely related to the net flux through the 
control volume, Eqs. (11) and (17) can be combined to yield

$$
\begin{aligned}
& \frac{\Gamma(2-\alpha)}{t^{1-\alpha}} \frac{\partial^{\alpha} \rho(\bar{x}, t) \theta(\bar{x}, t)}{(\partial t)^{\alpha}}=-\left[\frac{\Gamma\left(2-\beta_{1}\right)}{x_{1}^{1-\beta_{1}}}\left(\frac{\partial}{\partial x_{1}}\right)^{\beta_{1}}\right. \\
& \left(\rho q_{x_{1}}(\bar{x} ; t)\right)+\frac{\Gamma\left(2-\beta_{2}\right)}{x_{2}^{1-\beta_{2}}}\left(\frac{\partial}{\partial x_{2}}\right)^{\beta_{2}}\left(\rho q_{x_{2}}(\bar{x} ; t)\right) \\
& \left.+\frac{\Gamma\left(2-\beta_{3}\right)}{x_{3}^{1-\beta_{3}}}\left(\frac{\partial}{\partial x_{3}}\right)^{\beta_{3}}\left(\rho q_{x_{3}}(\bar{x} ; t)\right)\right], \\
& \frac{\Gamma(2-\alpha)}{t^{1-\alpha}} \frac{\partial^{\alpha} \rho(\bar{x}, t) \theta(\bar{x}, t)}{(\partial t)^{\alpha}}=-\sum_{i=1}^{3} \frac{\Gamma\left(2-\beta_{i}\right)}{x_{i}^{1-\beta_{i}}}\left(\frac{\partial}{\partial x_{i}}\right)^{\beta_{i}} \\
& \left(\rho(\bar{x} ; t) q_{x_{i}}(\bar{x} ; t)\right)
\end{aligned}
$$

as the fractional continuity equation of transient soil water flow in multi-fractional space of a generally anisotropic soil medium in fractional time.

If one further assumes an incompressible soil medium with constant density, then the fractional soil water flow continuity Eq. (18) simplifies further to

$$
\begin{gathered}
\frac{\Gamma(2-\alpha)}{t^{1-\alpha}} \frac{\partial^{\alpha} \theta(\bar{x}, t)}{(\partial t)^{\alpha}}=-\sum_{i=1}^{3} \frac{\Gamma\left(2-\beta_{i}\right)}{x_{i}^{1-\beta_{i}}}\left(\frac{\partial}{\partial x_{i}}\right)^{\beta_{i}} \\
\left(q_{x_{i}}(\bar{x} ; t)\right), 0<\alpha, \beta_{1}, \beta_{2}, \beta_{3}<1 ; \bar{x}=\left(x_{1}, x_{2}, x_{3}\right) .
\end{gathered}
$$

In the following, Eq. (19) will be used as the fractional continuity equation for soil water flow for further study.

Performing a dimensional analysis of Eq. (19), one obtains

$$
\frac{1}{T^{1-\alpha}} \cdot \frac{1}{T^{\alpha}}=\frac{1}{L^{1-\beta_{i}}} \frac{1}{L^{\beta_{i}}} \frac{L}{T}=\frac{1}{T},
$$

where $L$ denotes length and $T$ denotes time. Hence, Eq. (20) shows the dimensional consistency of the LHS and RHS of the continuity Eq. (19) for transient soil water flow in multifractional space and fractional time.

Podlubny (1999) has shown that for $n-1<\alpha, \beta_{i}<n$, where $n$ is any positive integer, as $\alpha$ and $\beta_{i} \rightarrow n$, the Caputo fractional derivative of a function $f(y)$ to order $\alpha$ or $\beta_{i}$ $(i=1,2,3)$ becomes the conventional $n$th derivative of the function $f(y)$. Therefore, specializing Podlubny's (1999) result to $n=1$, for $\alpha$ and $\beta_{i} \rightarrow 1(i=1,2,3)$, the continuity Eq. (19) reduces to

$$
\frac{\partial \theta(\bar{x}, t)}{\partial t}=-\sum_{i=1}^{3} \frac{\partial}{\partial x_{i}}\left(q_{x_{i}}(\bar{x} ; t)\right),
$$

which is the conventional continuity equation for soil water flow.

\section{An equation for soil water flux (specific discharge) in fractional time-space}

The experiments of Darcy (1856) showed that the specific discharge $q_{i}$ is directly proportional to the change in hy- draulic head, $\Delta h=h\left(x_{i}\right)-h\left(x_{i}-\Delta x\right), i=1,2,3$, and is inversely proportional to the spatial displacement in any direction $i, \Delta x_{i}=x_{i}-\left(x_{i}-\Delta x_{i}\right), i=1,2,3$ (Freeze and Cherry, 1979). Hence, one can express the Darcy law in integer timespace as

$q_{x_{i}} \Delta x_{i}=-K_{i} \Delta h_{i}, \quad i=1,2,3$,

where $K_{i}=K_{i}(\bar{x})$ denotes the hydraulic conductivity in the $i$ th spatial direction $(i=1,2,3)$, and the negative sign on the RHS of Eq. (22) is due to soil water flow being in the direction of decreasing hydraulic head.

In Eq. (22), using the $\beta$-order approximation to a function around $x-\Delta x$ in Eq. (5) to $\beta_{i}$-order $(i=1,2,3)$ yields, with $D_{0}^{\beta_{i}} h=\frac{\partial^{\beta_{i}} h}{\left(\partial x_{i}\right)^{\beta_{i}}}$,

$\Delta h_{i}=\frac{\left(\Delta x_{i}\right)^{\beta_{i}}}{\Gamma\left(\beta_{i}+1\right)} \frac{\partial^{\beta_{i}} h}{\left(\partial x_{i}\right)^{\beta_{i}}}, \quad i=1,2,3$,

where the lower limit in the integral of the Caputo derivative is again taken at zero. Combining Eqs. (10) and (23) with Eq. (22) yields,

$q_{i}\left[\frac{x_{i}^{1-\beta_{i}}}{\Gamma\left(2-\beta_{i}\right)}\right]=-K_{i}\left[\frac{\partial^{\beta_{i}} h}{\left(\partial x_{i}\right)^{\beta_{i}}}\right], \quad i=1,2,3$.

Expressing Eq. (24) for the specific discharge $q_{i}$, one obtains

$q_{i}(\bar{x}, t)=-K_{i}(\bar{x}) \frac{\Gamma\left(2-\beta_{i}\right)}{x_{i}^{1-\beta_{i}}} \frac{\partial^{\beta_{i}} h}{\left(\partial x_{i}\right)^{\beta_{i}}}, \quad i=1,2,3$

as the equation of water flux through anisotropic soil media in multi-fractional multi-dimensional space.

Performing a dimensional analysis on Eq. (25), one obtains

$$
\begin{aligned}
{\left[q_{i}(\bar{x}, t)\right] } & =L / T \text { and }\left[K_{i}(\bar{x}) \frac{\Gamma\left(2-\beta_{i}\right)}{x_{i}^{1-\beta_{i}}} \frac{\partial^{\beta_{i}} h}{\left(\partial x_{i}\right)^{\beta_{i}}}\right] \\
& =\frac{L}{T} \frac{L}{L^{1-\beta_{i}} L^{\beta_{i}}}=\frac{L}{T},
\end{aligned}
$$

which establishes the dimensional consistency of Eq. (25) as the fractional equation for soil water flux. Furthermore, it is well known that for unsaturated soil water flow, the hydraulic conductivity is function of the volumetric soil water content $\theta$ and of spatial location (Freeze and Cherry, 1979). In fact, $K_{i}$ may be expressed in terms of the saturated hydraulic conductivity $K_{\mathrm{s}}$ and the relative hydraulic conductivity $K_{\mathrm{r}}(\theta)$ as

$$
K_{i}(\bar{x}, \theta)=K_{\mathrm{s}, i}(\bar{x}) K_{\mathrm{r}}(\theta) .
$$

Hence, the equation of soil water flux (specific discharge) in multi-dimensional, multi-fractional anisotropic soil space may be expressed as

$q_{i}(\bar{x}, t)=-K_{i}(\bar{x}, \theta) \frac{\Gamma\left(2-\beta_{i}\right)}{x_{i}^{1-\beta_{i}}} \frac{\partial^{\beta_{i}} h(\bar{x}, t)}{\left(\partial x_{i}\right)^{\beta_{i}}}, i=1,2,3$. 
Equation (28) is dimensionally consistent in that both the LHS and RHS of the equation have the unit $L / T$.

As noted above, Podlubny (1999) has shown that for $n-1<\beta_{i}<n(i=1,2,3)$, where $n$ is any positive integer, as $\beta_{i} \rightarrow n$, the Caputo fractional derivative of a function $f(y)$ to order $\beta_{i}(i=1,2,3)$ becomes the conventional $n$th derivative of the function $f(y)$. Therefore, specializing Podlubny's (1999) result to $n=1$, for $\beta_{i} \rightarrow 1(i=1,2,3)$, the fractional soil water flux Eq. (28) becomes

$q_{i}(\bar{x}, t)=-K_{i}(\bar{x}, \theta) \frac{\partial h(\bar{x}, t)}{\partial x_{i}}, \quad i=1,2,3$,

which is the conventional Darcy equation for soil water flux. As such the derived fractional soil water flux Eq. (28) is consistent with the conventional Darcy equation for the integer power case.

\section{Governing equation of transient soil water flow in multi-dimensional fractional soil space and fractional time}

Combining the fractional continuity Eq. (19) with the fractional soil water flux Eq. (28) yields

$$
\begin{aligned}
& \frac{\Gamma(2-\alpha)}{t^{1-\alpha}} \frac{\partial^{\alpha} \theta(\bar{x}, t)}{(\partial t)^{\alpha}}=\sum_{i=1}^{3} \frac{\Gamma\left(2-\beta_{i}\right)}{x_{i}^{1-\beta_{i}}}\left(\frac{\partial}{\partial x_{i}}\right)^{\beta_{i}} \\
& \quad\left(K_{i}(\bar{x}, \theta) \frac{\Gamma\left(2-\beta_{i}\right)}{x_{i}^{1-\beta_{i}}} \frac{\partial^{\beta_{i}} h(\bar{x}, t)}{\left(\partial x_{i}\right)^{\beta_{i}}}\right) \\
& \text { for } 0<\alpha, \beta_{1}, \beta_{2}, \beta_{3}<1 ; \bar{x}=\left(x_{1}, x_{2}, x_{3}\right) .
\end{aligned}
$$

Since $K_{i}(\bar{x}, \theta)=K_{\mathrm{s}, i}(\bar{x}) \mathrm{K}_{\mathrm{r}}(\theta)$ one obtains

$$
\begin{gathered}
\frac{\Gamma(2-\alpha)}{t^{1-\alpha}} \frac{\partial^{\alpha} \theta(\bar{x}, t)}{(\partial t)^{\alpha}}=\sum_{i=1}^{3} \frac{\Gamma\left(2-\beta_{i}\right)}{x_{i}^{1-\beta_{i}}}\left(\frac{\partial}{\partial x_{i}}\right)^{\beta_{i}} \\
\left(K_{\mathrm{s}, i}(\bar{x}) \mathrm{K}_{\mathrm{r}}(\theta) \frac{\Gamma\left(2-\beta_{i}\right)}{x_{i}^{1-\beta_{i}}} \frac{\partial^{\beta_{i}} h(\bar{x}, t)}{\left(\partial x_{i}\right)^{\beta_{i}}}\right) \\
\text { for } 0<\alpha, \beta_{1}, \beta_{2}, \beta_{3}<1 ; \bar{x}=\left(x_{1}, x_{2}, x_{3}\right)
\end{gathered}
$$

as the governing equation of transient soil water flow in anisotropic multi-dimensional fractional soil media and fractional time.

Meanwhile, the soil hydraulic head $\mathrm{h}$ is related to the elevation head $x_{3}$ and soil capillary pressure head $\psi$ by

$h=\psi(\theta)+x_{3}$.
Substituting Eq. (32) into Eq. (31) results in

$$
\begin{gathered}
\frac{\Gamma(2-\alpha)}{t^{1-\alpha}} \frac{\partial^{\alpha} \theta(\bar{x}, t)}{(\partial t)^{\alpha}}=\sum_{i=1}^{3} \frac{\Gamma\left(2-\beta_{i}\right)}{x_{i}^{1-\beta_{i}}}\left(\frac{\partial}{\partial x_{i}}\right)^{\beta_{i}} \\
\left(K_{\mathrm{S}, i}(\bar{x}) K_{\mathrm{r}}(\theta) \frac{\Gamma\left(2-\beta_{i}\right)}{x_{i}^{1-\beta_{i}}} \frac{\partial^{\beta_{i}}}{\left(\partial x_{i}\right)^{\beta_{i}}}\left(\psi(\theta)+x_{3}\right)\right) .
\end{gathered}
$$

With respect to the Caputo derivative:

$$
\frac{\partial^{\beta_{3}} x_{3}}{\left(\partial x_{3}\right)^{\beta_{3}}}=\frac{x_{3}^{1-\beta_{3}}}{\Gamma\left(2-\beta_{3}\right)} \text {. }
$$

Opening Eq. (33) further and introducing Eq. (34) yields

$$
\begin{gathered}
\frac{\Gamma(2-\alpha)}{t^{1-\alpha}} \frac{\partial^{\alpha} \theta(\bar{x}, t)}{(\partial t)^{\alpha}}=\sum_{i=1}^{3} \frac{\Gamma\left(2-\beta_{i}\right)}{x_{i}^{1-\beta_{i}}}\left(\frac{\partial}{\partial x_{i}}\right)^{\beta_{i}} \\
\left(K_{\mathrm{s}, i}(\bar{x}) \mathrm{K}_{\mathrm{r}}(\theta) \frac{\Gamma\left(2-\beta_{i}\right)}{x_{i}^{1-\beta_{i}}} \frac{\partial^{\beta_{i}} \psi(\theta)}{\left(\partial x_{i}\right)^{\beta_{i}}}\right) \\
+\frac{\Gamma\left(2-\beta_{3}\right)}{x_{3}^{1-\beta_{3}}}\left(\frac{\partial}{\partial x_{3}}\right)^{\beta_{3}}\left(K_{\mathrm{s}, 3}(\bar{x}) \mathrm{K}_{\mathrm{r}}(\theta)\right) ; \\
0<\alpha, \beta_{1}, \beta_{2}, \beta_{3}<1 ; \bar{x}=\left(x_{1}, x_{2}, x_{3}\right)
\end{gathered}
$$

as the governing equation of transient soil water flow in anisotropic multi-dimensional fractional media and fractional time. This governing equation may also be written as

$$
\begin{aligned}
& \frac{\partial^{\alpha} \theta(\bar{x}, t)}{(\partial t)^{\alpha}}=\sum_{i=1}^{3} \frac{1}{\Gamma(2-\alpha)} \frac{\left(\Gamma\left(2-\beta_{i}\right)\right)^{2}}{x_{i}^{1-\beta_{i}}}\left(\frac{\partial}{\partial x_{i}}\right)^{\beta_{i}} \\
& \left(K_{\mathrm{s}, i}(\bar{x}) K_{\mathrm{r}}(\theta) \frac{t^{1-\alpha}}{x_{i}^{1-\beta_{i}}} \frac{\partial^{\beta_{i}} \psi(\theta)}{\left(\partial x_{i}\right)^{\beta_{i}}}\right) \\
& \quad+\frac{1}{\Gamma(2-\alpha)} \frac{\Gamma\left(2-\beta_{3}\right)}{x_{3}^{1-\beta_{3}}}\left(\frac{\partial}{\partial x_{3}}\right)^{\beta_{3}}\left(t^{1-\alpha} K_{\mathrm{s}, 3}(\bar{x}) K_{\mathrm{r}}(\theta)\right) ; \\
& 0<\alpha, \beta_{1}, \beta_{2}, \beta_{3}<1 ; \bar{x}=\left(x_{1}, x_{2}, x_{3}\right) .
\end{aligned}
$$

As noted above, Podlubny (1999) has shown that for $n-$ $1<\alpha, \beta_{i}<n(i=1,2,3)$, where $n$ is any positive integer, as $\alpha$ and $\beta_{i} \rightarrow n$, the Caputo fractional derivative of a function $f(y)$ to order $\alpha$ or $\beta_{i}(i=1,2,3)$ becomes the conventional $n$th derivative of the function $f(y)$. Therefore, specializing Podlubny's (1999) result to $n=1$, for $\alpha$ and $\beta_{i} \rightarrow 1$ ( $i=1$, 2,3 ), the fractional governing Eq. (33) of soil water flow becomes

$$
\frac{\partial \theta(\bar{x}, t)}{\partial t}=\sum_{i=1}^{3} \frac{\partial}{\partial x_{i}}\left(K_{\mathrm{S}, i}(\bar{x}) \mathrm{K}_{\mathrm{r}}(\theta) \frac{\partial}{\partial x_{i}}\left(\psi(\theta)+x_{3}\right)\right),
$$

which is the conventional Richards equation for transient soil water flow.

With respect to dimensional consistency, one may note that both sides of the fractional governing Eqs. (33) or (35) for transient soil water flow have the unit $1 / T$. Meanwhile, both sides of Eq. (36) have the unit $1 / T^{\alpha}$. Hence, these fractional equations are dimensionally consistent. 


\section{Fractional governing equation of transient soil water flow in the vertical direction}

In the case of vertical transient unsaturated flow for the infiltration process in soils in fractional time-space, Eq. (35) reduces further to

$$
\begin{gathered}
\frac{\Gamma(2-\alpha)}{t^{1-\alpha}} \frac{\partial^{\alpha} \theta(\bar{x}, t)}{(\partial t)^{\alpha}}=\frac{\Gamma\left(2-\beta_{3}\right)}{x_{3}^{1-\beta_{3}}}\left(\frac{\partial}{\partial x_{3}}\right)^{\beta_{3}} \\
\left(K_{\mathrm{s}, 3}(\bar{x}) \mathrm{K}_{\mathrm{r}}(\theta) \frac{\Gamma\left(2-\beta_{3}\right)}{x_{3}^{1-\beta_{3}}} \frac{\partial^{\beta_{3}} \psi(\theta)}{\left(\partial x_{3}\right)^{\beta_{3}}}\right) \\
+\frac{\Gamma\left(2-\beta_{3}\right)}{x_{3}^{1-\beta_{3}}}\left(\frac{\partial}{\partial x_{3}}\right)^{\beta_{3}}\left(K_{\mathrm{s}, 3}(\bar{x}) K_{\mathrm{r}}(\theta)\right) ; \\
0<\alpha, \beta_{3}<1 ; \bar{x}=\left(x_{1}, x_{2}, x_{3}\right)
\end{gathered}
$$

as the governing equation. This governing equation for vertical transient soil water flow in fractional time-space can also be expressed as

$$
\begin{aligned}
& \frac{\partial^{\alpha} \theta(\bar{x}, t)}{(\partial t)^{\alpha}}=\frac{\Gamma\left(2-\beta_{3}\right)}{x_{3}^{1-\beta_{3}}}\left(\frac{\partial}{\partial x_{3}}\right)^{\beta_{3}} \\
& \left(K_{\mathrm{s}, 3}(\bar{x}) K_{\mathrm{r}}(\theta) \frac{\Gamma\left(2-\beta_{3}\right)}{\Gamma(2-\alpha)} \frac{t^{1-\alpha}}{x_{3}^{1-\beta_{3}}} \frac{\partial^{\beta_{3}} \psi(\theta)}{\left(\partial x_{3}\right)^{\beta_{3}}}\right) \\
& +\frac{1}{\Gamma(2-\alpha)} \frac{\Gamma\left(2-\beta_{3}\right)}{x_{3}^{1-\beta_{3}}}\left(\frac{\partial}{\partial x_{3}}\right)^{\beta_{3}}\left(t^{1-\alpha} K_{\mathrm{s}, 3}(\bar{x}) K_{\mathrm{r}}(\theta)\right) \\
& 0<\alpha, \beta_{3}<1 ; \bar{x}=\left(x_{1}, x_{2}, x_{3}\right) .
\end{aligned}
$$

As in the integer case of Richards Eq. (37), Eqs. (35), (36), (38) and (39) have both the hydraulic conductivity $\mathrm{K}$ and the capillary pressure head $\psi$ as functions of the soil volumetric water content $\theta$. As such, characteristic soil water relationships, such as those given by Brooks and Corey (1964), may be utilized to obtain an explicit form of the governing equation of transient, unsaturated soil water flow in fractional time-space, as explained in the following.

\section{Soil water content-based explicit form of the governing equation of transient soil water flow in fractional time-space}

One can utilize the Brooks and Corey (1964) formula for the soil characteristic relationship between the capillary soil water pressure head $\psi$ and the soil water content $\theta$ as follows:

$\psi(\theta)=\psi_{\mathrm{b}} \theta_{\mathrm{e}}^{1 / \lambda}\left(\theta-\theta_{r}\right)^{-1 / \lambda}$,

where $\psi_{\mathrm{b}}$ is the air entry pressure head (bubbling pressure), $\theta_{\mathrm{e}}=\left(\theta_{\mathrm{s}}-\theta_{\mathrm{r}}\right)$ is the effective porosity, $\theta_{\mathrm{s}}$ is the saturation volumetric soil water content, $\theta_{\mathrm{r}}$ is the residual water content, and $\lambda$ is the pore size distribution index. Therefore, the $\beta_{i}$ order Caputo fractional derivative of the capillary pressure head $\psi$ with respect to $x_{i}$ in the interval $\left(0, x_{i}\right)$ may be expressed in terms of the Brooks-Corey relationship (Eq. 40) as (Podlubny, 1999; Odibat and Shawagfeh, 2007)

$$
\begin{gathered}
\frac{\partial^{\beta_{i}} \psi(\theta)}{\left(\partial x_{i}\right)^{\beta_{i}}}=\frac{\psi_{\mathrm{b}} \theta_{\mathrm{e}}^{1 / \lambda}}{\Gamma\left(1-\beta_{i}\right)} \int_{0}^{x_{i}}\left(\frac{\partial}{\partial \xi_{i}}\left(\theta-\theta_{\mathrm{r}}\right)^{-1 / \lambda}\right) \\
\left(x_{i}-\xi_{i}\right)^{-\beta_{i}} \mathrm{~d} \xi_{i}=\psi_{\mathrm{b}} \theta_{\mathrm{e}}^{1 / \lambda} \frac{\partial^{\beta_{i}}\left(\theta-\theta_{\mathrm{r}}\right)^{-1 / \lambda}}{\left(\partial x_{i}\right)^{\beta_{i}}} .
\end{gathered}
$$

Under the Brooks and Corey (1964) relationship between the hydraulic conductivity and the volumetric soil water content, the relative hydraulic conductivity $K_{\mathrm{r}}(\theta)$ is expressed as

$$
K_{\mathrm{r}}(\theta)=\theta_{\mathrm{e}}^{-3-2 / \lambda}\left(\theta-\theta_{\mathrm{r}}\right)^{3+2 / \lambda}
$$

and using expression (42) within $K_{i}(\bar{x}, \theta)=K_{\mathrm{S}, i}(\bar{x}) K_{\mathrm{r}}(\theta)$, the $\beta_{i}$-order fractional Caputo derivative of $K_{i}(\bar{x}, \theta)$ with respect to $x_{i}$ in the interval $\left(0, x_{i}\right)$ may be expressed as

$$
\begin{aligned}
& \frac{\partial^{\beta_{i}} K_{\mathrm{s}, i}(\bar{x}) K_{\mathrm{r}}(\theta)}{\left(\partial x_{i}\right)^{\beta_{i}}}=\theta_{\mathrm{e}}^{-3-2 / \lambda} \frac{\partial^{\beta_{i}}\left(K_{\mathrm{s}, i}(\bar{x})\left(\theta-\theta_{\mathrm{r}}\right)^{3+2 / \lambda}\right)}{\left(\partial x_{i}\right)^{\beta_{i}}}, \\
& \quad i=1,2,3 .
\end{aligned}
$$

Substituting Eqs. (41) and (43) into Eq. (35) results in an explicit form of the governing equation of transient soil water flow in anisotropic multi-dimensional fractional soil space and fractional time in terms of the volumetric water content $\theta$ as

$$
\begin{aligned}
& \frac{\Gamma(2-\alpha)}{t^{1-\alpha}} \frac{\partial^{\alpha} \theta(\bar{x}, t)}{(\partial t)^{\alpha}}=\sum_{i=1}^{3} \psi_{\mathrm{b}} \theta_{\mathrm{e}}^{-3-1 / \lambda} \frac{\left(\Gamma\left(2-\beta_{i}\right)\right)^{2}}{x_{i}^{1-\beta_{i}}}\left(\frac{\partial}{\partial x_{i}}\right)^{\beta_{i}} \\
& \left(K_{\mathrm{s}, i}(\bar{x}) \frac{\left(\theta-\theta_{\mathrm{r}}\right)^{3+2 / \lambda}}{x_{i}^{1-\beta_{i}}} \frac{\partial^{\beta_{i}}\left(\theta-\theta_{\mathrm{r}}\right)^{-1 / \lambda}}{\left(\partial x_{i}\right)^{\beta_{i}}}\right) \\
& +\theta_{\mathrm{e}}^{-3-2 / \lambda} \frac{\Gamma\left(2-\beta_{3}\right)}{x_{3}^{1-\beta_{3}}}\left(\frac{\partial}{\partial x_{3}}\right)^{\beta_{3}}\left(K_{\mathrm{s}, 3}(\bar{x})\left(\theta-\theta_{\mathrm{r}}\right)^{3+2 / \lambda}\right) ; \\
& 0<\alpha, \beta_{1}, \beta_{2}, \beta_{3}<1
\end{aligned}
$$

in terms of the Brooks-Corey soil water characteristics relationships. This governing equation can also be expressed as

$$
\begin{gathered}
\frac{\partial^{\alpha} \theta(\bar{x}, t)}{(\partial t)^{\alpha}}=\sum_{i=1}^{3} \psi_{\mathrm{b}} \theta_{\mathrm{e}}^{-3-1 / \lambda} \frac{\left(\Gamma\left(2-\beta_{i}\right)\right)^{2}}{\Gamma(2-\alpha) x_{i}^{1-\beta_{i}}}\left(\frac{\partial}{\partial x_{i}}\right)^{\beta_{i}} \\
\left(K_{\mathrm{s}, i}(\bar{x})\left(\theta-\theta_{\mathrm{r}}\right)^{3+2 / \lambda} \frac{t^{1-\alpha}}{x_{i}^{1-\beta_{i}}} \frac{\partial^{\beta_{i}}\left(\theta-\theta_{\mathrm{r}}\right)^{-1 / \lambda}}{\left(\partial x_{i}\right)^{\beta_{i}}}\right) \\
+\theta_{\mathrm{e}}^{-3-2 / \lambda} \frac{\Gamma\left(2-\beta_{3}\right)}{\Gamma(2-\alpha) x_{3}^{1-\beta_{3}}}\left(\frac{\partial}{\partial x_{3}}\right)^{\beta_{3}} \\
\left(t^{1-\alpha} K_{\mathrm{s}, 3}(\bar{x})\left(\theta-\theta_{\mathrm{r}}\right)^{3+2 / \lambda}\right) ; 0<\alpha, \beta_{1}, \beta_{2}, \beta_{3}<1 .
\end{gathered}
$$

Upon dimensional analysis of Eq. (44) one can see that it is dimensionally consistent since both of its sides have the 
unit of $1 / T$, where $T$ denotes time. Meanwhile, Eq. (45) is also dimensionally consistent with both sides of the equation having the unit $1 / T^{\alpha}$.

Specializing Eq. (45) to only the vertical direction, the governing equation of transient soil water flow in the vertical direction in fractional space-time may be expressed as

$$
\begin{gathered}
\frac{\partial^{\alpha} \theta(\bar{x}, t)}{(\partial t)^{\alpha}}=\psi_{\mathrm{b}} \theta_{\mathrm{e}}^{-3-1 / \lambda} \frac{\left(\Gamma\left(2-\beta_{3}\right)\right)^{2}}{\Gamma(2-\alpha) x_{3}^{1-\beta_{3}}}\left(\frac{\partial}{\partial x_{3}}\right)^{\beta_{3}} \\
\left(K_{\mathrm{s}, 3}(\bar{x})\left(\theta-\theta_{\mathrm{r}}\right)^{3+2 / \lambda} \frac{t^{1-\alpha}}{x_{3}^{1-\beta_{3}}} \frac{\partial^{\beta_{3}}\left(\theta-\theta_{\mathrm{r}}\right)^{-1 / \lambda}}{\left(\partial x_{3}\right)^{\beta_{3}}}\right) \\
+\theta_{\mathrm{e}}^{-3-2 / \lambda} \frac{\Gamma\left(2-\beta_{3}\right)}{\Gamma(2-\alpha) x_{3}^{1-\beta_{3}}}\left(\frac{\partial}{\partial x_{3}}\right)^{\beta_{3}} \\
\left(t^{1-\alpha} K_{\mathrm{s}, 3}(\bar{x})\left(\theta-\theta_{\mathrm{r}}\right)^{3+2 / \lambda}\right) ; 0<\alpha, \beta_{3}<1 .
\end{gathered}
$$

Upon dimensional analysis of Eq. (46) one can find that both sides of this equation have the unit of $1 / T^{\alpha}$, where $T$ denotes time. Hence, the fractional equation of vertical transient soil water flow, in its explicit form, is dimensionally consistent.

Finally, specializing Eq. (45) to only the horizontal directions, the governing equation of transient soil water flow in the horizontal directions in fractional space-time may be expressed as

$$
\begin{aligned}
& \frac{\partial^{\alpha} \theta(\bar{x}, t)}{(\partial t)^{\alpha}}=\sum_{i=1}^{2} \psi_{\mathrm{b}} \theta_{\mathrm{e}}^{-3-1 / \lambda} \frac{\left(\Gamma\left(2-\beta_{i}\right)\right)^{2}}{\Gamma(2-\alpha) x_{i}^{1-\beta_{i}}}\left(\frac{\partial}{\partial x_{i}}\right)^{\beta_{i}} \\
& \left(K_{\mathrm{s}, i}(\bar{x})\left(\theta-\theta_{\mathrm{r}}\right)^{3+2 / \lambda} \frac{t^{1-\alpha}}{x_{i}^{1-\beta_{i}}} \frac{\partial^{\beta_{i}}\left(\theta-\theta_{\mathrm{r}}\right)^{-1 / \lambda}}{\left(\partial x_{i}\right)^{\beta_{i}}}\right) \\
& 0<\alpha, \beta_{1}, \beta_{2}<1 .
\end{aligned}
$$

Upon dimensional analysis of Eq. (47) one can find that both sides of this equation have the unit of $1 / T^{\alpha}$, where $T$ denotes time. Hence, the fractional equation of horizontal transient soil water flow, in its explicit form, is dimensionally consistent.

\section{Physical framework for the developed time-space fractional governing equations of soil water flow}

In parallel to the conventional governing equations of soil water flow processes (Freeze and Cherry, 1979; Bear, 1979), the corresponding governing equations of the soil water flow processes in fractional time-space must have certain properties. (i) The fractional governing equations must be purely differential equations, containing only differential operators, and no difference operators. (ii) They must be prognostic equations. That is, they are solved from the initial conditions and boundary conditions in order to describe the evolution of the flow field in time and space. As such, from the outset the form of the governing equation must be known in its entirety. Once its physical parameters (such as the saturated hydraulic conductivity, etc.) are estimated, the governing equation is fixed throughout the simulation time and the simulation space for the simulation of the soil water flow in question. (iii) These equations must be dimensionally consistent. (iv) The fractional governing equations of soil water flow with fractional powers must converge to the corresponding conventional governing equations with integer powers as the fractional powers approach the corresponding integer powers.

However, a distinct difference of the fractional governing equations of soil water flow from the corresponding conventional equations is that they are based on fractional derivatives which are nonlocal. Being nonlocal, the fractional governing equations of soil water flow have the potential to account for the effect of the initial conditions on the soil water flow for long times, and for the effect of the upstream boundary conditions on the flow for long distances from the upstream boundary. The physical meaning of the fractional governing equation may be explained most easily in the case of vertical soil water flow. In the context of upstreamto-downstream vertical soil water flow from the soil surface downward, in the integer form of the soil water flow mass conservation equation (the conventional equation) the time rate of change of mass within a control volume grid $(x-\Delta x, x)$ is determined by the mass flux coming from the upstream neighbor grid $(x-2 \Delta x, x-\Delta x)$ into $(x-\Delta x, x)$, and the mass flux that is moving from the control volume grid $(x-\Delta x, x)$ to the downstream neighbor grid $(x, x+\Delta x)$. This framework holds also for the soil water flow in the two horizontal directions. As such, the mass evolution in the case of the integer governing equation of soil water flow is local (at the scale of the specific computational grid), due to interaction only among neighboring computational grids. On the other hand, in the case of the fractional governing equation of mass of vertical upstream-to-downstream soil water flow from the soil surface downward, we deal with the Caputo fractional derivative

$$
\frac{\partial^{\beta} f}{\left(\partial x_{3}\right)^{\beta}}=D_{0}^{\beta} f\left(x_{3}\right)
$$

defined by

$$
\begin{aligned}
& D_{0}^{\beta} f\left(x_{3}\right)=\frac{1}{\Gamma(1-\beta)} \int_{0}^{x_{3}} \frac{f^{\prime}(\xi)}{\left(x_{3}-\xi\right)^{\beta}} \mathrm{d} \xi \\
& 0<\beta<1, x_{3} \geq 0 .
\end{aligned}
$$

As such, each local integer derivative $f^{\prime}(\xi)$ at each depth $\xi$ in the interval $\left(0, x_{3}\right)$ contributes to the Caputo fractional derivative of the interval $\left(0, x_{3}\right)$ with weight $\left(x_{3}-\xi\right)^{-\beta}$. Within this framework, for example, in the case of onedimensional downward vertical soil water flow in fractional time-space, the effect of the upstream boundary condition at depth zero is still accounted for at any depth $x_{3}$ below the 
soil surface by means of the fractional spatial derivatives that appear in the corresponding governing equation (Eq. 39 or Eq. 46 above). It also follows from Eq. (49) that this memory effect is modulated by the value of the fractional power $\beta$. This is also the case in the time dimension where the effect of the initial condition at time zero is accounted for at any time $t$ after the initial time. Also, the effects of the local derivatives at each time $s(0 \leq s \leq t)$ on the Caputo derivative of the interval $(0, t)$ are accounted for with the weights $(t-s)^{-\alpha}$. Hence, the fractional governing equations of soil water flow are nonlocal, and, as such, can quantify the influence of the initial and boundary conditions on the flow process more effectively than the corresponding conventional governing equations that are local.

Referring to Eq. (4) above, it is necessary to take the upper limit value of the Caputo derivative at " $x$ " in the mean value representation of a function in terms of the fractional Caputo derivative (Usero, 2008; Li et al., 2009; Odibat and Shawagfeh, 2007) in order to have the governing equations, based on this approximation, become prognostic equations that shall be known from the outset of model simulation for the whole time-space modeling domain. Then referring to Eq. (5) above, in order to have the governing equations to have purely differential forms (with only the differential operators (and no difference operators) existing in these equations), it is necessary to establish an analytical relationship between $\Delta x$ and $(\Delta x)^{\beta}$. This is possible by taking the origin of the Caputo derivative in Eq. (5) at zero (the upstream boundary location in space or initial time location in time). Otherwise, when one evaluates the Caputo derivative of the function $x$ at the integral limits $(x-\Delta x, x)$, one ends up with a fractional binomial expansion that has infinite number of terms, which prevents an analytical relationship between $\Delta x$ and $(\Delta x)^{\beta}$. This is also the case for the time dimension. The Caputo derivative of the function $t$ in the time dimension must again be evaluated at the lower limit of the integral set at the initial time zero in order to obtain purely differential operators for the evolution in time for the governing equations. It is also important to note that under these approximations, the resulting governing equations are all dimensionally consistent, and all the resulting fractional governing equations converge to their corresponding conventional counterparts with integer powers as their fractional powers approach unity.

\section{Discussion and conclusion}

The governing equations that were developed in this study are for the fractional time dimension and for multidimensional fractional space that represents the fractal spatial structure of a soil field. If one were to simplify the developed theory above to only fractional time but integer-space soil fields, then the developed equations would simplify substantially. The governing Eq. (36) of transient soil water flow in anisotropic multi-dimensional fractional soil media in fractional time would simplify to (with $\beta_{i}=1, i=1,2,3$ )

$$
\begin{aligned}
& \frac{\partial^{\alpha} \theta(\bar{x}, t)}{(\partial t)^{\alpha}}=\sum_{i=1}^{3} \frac{1}{\Gamma(2-\alpha)} \frac{\partial}{\partial x_{i}}\left(K_{\mathrm{s}, i}(\bar{x}) K_{\mathrm{r}}(\theta) t^{1-\alpha} \frac{\partial \psi(\theta)}{\partial x_{i}}\right) \\
& +\frac{1}{\Gamma(2-\alpha)} \frac{\partial}{\partial x_{3}}\left(t^{1-\alpha} K_{\mathrm{s}, 3}(\bar{x}) K_{\mathrm{r}}(\theta)\right) \\
& 0<\alpha<1 ; \bar{x}=\left(x_{1}, x_{2}, x_{3}\right)
\end{aligned}
$$

for the governing equation of transient soil water flow in integer multi-dimensional soil media and in fractional time. In terms of the Brooks-Corey soil characteristic relationships, the explicit governing equation of transient soil water flow in integer multi-dimensional soil space and in fractional time is obtained from the simplification of Eq. (45) as (with $\beta_{i}=1$, $i=1,2,3)$

$$
\begin{aligned}
& \frac{\partial^{\alpha} \theta(\bar{x}, t)}{(\partial t)^{\alpha}}=\sum_{i=1}^{3}-1 / \lambda \psi_{\mathrm{b}} \theta_{\mathrm{e}}^{-3-1 / \lambda} \frac{1}{\Gamma(2-\alpha)} \frac{\partial}{\partial x_{i}} \\
& \left(t^{1-\alpha} K_{\mathrm{s}, i}(\bar{x})\left(\theta-\theta_{\mathrm{r}}\right)^{2+1 / \lambda} \frac{\partial \theta}{\partial x_{i}}\right) \\
& \quad+\theta_{\mathrm{e}}^{-3-2 / \lambda} \frac{1}{\Gamma(2-\alpha)} \frac{\partial}{\partial x_{3}}\left(t^{1-\alpha} K_{\mathrm{s}, 3}(\bar{x})\left(\theta-\theta_{\mathrm{r}}\right)^{3+2 / \lambda}\right) \\
& 0<\alpha<1 ; \bar{x}=\left(x_{1}, x_{2}, x_{3}\right) .
\end{aligned}
$$

As mentioned before, Guerrini and Swartzendruber (1992) and El-Abd and Milczarek (2004), in their explanation of the anomalous behavior of the diffusivity coefficient in their experiments, have proposed that the diffusivity coefficient in the diffusion-based formulation of the Richards equation of soil water flow must depend not only on the water content but also on time. Hence, they formulated this diffusivity coefficient $D$ as $D=D(\theta, t)=E(\theta) t^{m}$, where $E$ is a function of water content $\theta$ while $\mathrm{m}$ is a power value. This formulation proved to be successful in modeling various experimental data on horizontal soil water flow. If one were to formulate the diffusivity $D_{i}(\theta, t)$ in the explicit governing Eq. (51) of transient soil water flow in fractional time and in anisotropic multi-dimensional integer soil space as

$D_{\mathrm{i}}(\theta, t)=K_{\mathrm{s}, i}(\bar{x})\left(\theta-\theta_{\mathrm{r}}\right)^{2+1 / \lambda} t^{1-\alpha}, \quad i=1,2,3$,

this diffusivity coefficient is in the same form as the diffusivity coefficient $D(\theta, t)=E(\theta) t^{m}$ that was formulated by Guerrini and Swartzendruber (1992) and El-Abd and Milczarek (2004) based on experimental observations. As such, within the framework of Brooks-Corey soil water relationships, the explicit governing equations that were developed in this study for the transient soil water flow in multidimensional fractional soil media and fractional time, when simplified to integer soil space, are consistent with the experimental observations of Guerrini and Swartzendruber (1992) and El-Abd and Milczarek (2004) when their power value $m=1-\alpha$.

Sun et al. (2013) conjectured that the time-dependent diffusivity $D(\theta, t)=E(\theta) t^{m}$ (for a fractional value of $m$ ) due 
to Guerrini and Swartzendruber (1992) and El-Abd and Milczarek (2004), in the conventional Richards equation can be expressed essentially by representing the conventional integer derivative of the soil water content with respect to time by a product of the fractional time derivative of the soil water content and a fractional power of time (Sun et al., 2013, Eq. 12), that is, $\frac{\partial \theta(\bar{x}, t)}{\partial t}=\frac{C}{t^{1-\alpha}} \frac{\partial^{\alpha} \theta(\bar{x}, t)}{(\partial t)^{\alpha}}$, where $C$ denotes a constant. In order to examine the conjecture of Sun et al. (2013), one can re-write the explicit governing Eq. (51) for soil water flow in integer space but fractional time in equivalent form as

$$
\begin{aligned}
& \frac{\Gamma(2-\alpha)}{t^{1-\alpha}} \frac{\partial^{\alpha} \theta(\bar{x}, t)}{(\partial t)^{\alpha}}=\sum_{i=1}^{3}-1 / \lambda \psi_{\mathrm{b}} \theta_{\mathrm{e}}^{-3-1 / \lambda} \frac{\partial}{\partial x_{i}} \\
& \left(K_{\mathrm{s}, i}(\bar{x})\left(\theta-\theta_{\mathrm{r}}\right)^{2+1 / \lambda} \frac{\partial \theta}{\partial x_{i}}\right)+\theta_{\mathrm{e}}^{-3-2 / \lambda} \frac{\partial}{\partial x_{3}} \\
& \left(K_{\mathrm{s}, 3}(\bar{x})\left(\theta-\theta_{\mathrm{r}}\right)^{3+2 / \lambda}\right) ; 0<\alpha<1 ; \bar{x}=\left(x_{1}, x_{2}, x_{3}\right) .
\end{aligned}
$$

Equation (53) shows that the fractional soil water flow Eq. (51) which accounts for the time-dependent diffusivity expression of Guerrini and Swartzendruber (1992) and ElAbd and Milczarek (2004) does have an equivalent form where the integer time derivative of the soil water content in the conventional Richards equation is replaced by a product of the fractional time derivative of the soil water content and a fractional power of time, thereby supporting Sun et al.'s (2013) conjecture, although in this study the fractional derivative is defined in the Caputo sense while in Sun et al. (2013) the fractional derivative is defined with respect to a fractal ruler in time.

In conclusion, in this study first a dimensionally consistent continuity equation for soil water flow in multi-fractional, multi-dimensional space and fractional time was developed. For the motion equation of soil water flow, or the equation of water flux within the soil matrix in multi-fractional multi-dimensional space and fractional time, a dimensionally consistent equation was also developed. From the combination of the fractional continuity and motion equations, the governing equation of transient soil water flow in multifractional, multi-dimensional space and fractional time was then obtained. It is shown that this equation approaches the conventional Richards equation as the fractional derivative powers approach integer values. Then by the introduction of the Brooks-Corey constitutive relationships for soil water (Brooks and Corey, 1964) into the fractional transient soil water flow equation, an explicit form of the equation was obtained in multi-dimensional, multi-fractional space and fractional time. Finally, the governing fractional equation was specialized to the cases of vertical soil water flow and horizontal soil water flow in fractional time-space. It is shown that the developed governing equations, in their fractional time but integer space forms, show behavior consistent with the previous experimental observations concerning the diffusive behavior of soil water flow.
Competing interests. The authors declare that they have no conflict of interest.

Edited by: Y. Chen

Reviewed by: T. Yamada and one anonymous referee

\section{References}

Baumer, B. and Meerschaert, M. M.: Fractional diffusion with two time scales, Physica A, 373, 237-251, 2007.

Baumer, B., Benson, D., and Meerschaert, M. M.: Advection and dispersion in time and space, Physica A, 350, 245-262, 2005.

Bear, J.: Hydraulics of groundwater, McGraw-Hill Inc., New York, 1979.

Benson, D. A., Wheatcraft, S. W., and Meerschaert, M. M.: Application of a fractional advection-dispersion equation, Water Resour. Res., 36, 1403-1412, 2000a.

Benson, D. A., Wheatcraft, S. W., and Meerschaert, M. M.: The fractional-order governing equation of Levy motion, Water Resour. Res., 36, 1413-1423, 2000b.

Brooks, R. H. and Corey, A. T.: Hydraulic properties of porous media, Hydrol. Pap. no. 3, Colorado State University, Fort Collins, CO, 1964.

Cushman, J. H., O’Malley, D., and Park, M.: Anomalous diffusion as modeled by a nonstationary extension of Brownian motion, Phys. Rev. E., 79, 032101, doi:10.1103/PhysRevE.79.032101, 2009.

Darcy, H.: Les Fontaines Publiques de la Ville de Dijon, Dalmont, Paris, 1856.

El-Abd, A. E.-G. and Milczarek, J. J..: Neutron radiography study of water absorption in porous building materials: anomalous diffusion analysis, J. Phys. D Appl. Phys., 37, 2305-2513, 2004.

Ferguson, H. and Gardner, W. H.: Diffusion theory applied to water flow data obtained using gamma ray absorption, Soil Sci. Soc. Am. Pro., 27, 243-246, 1963.

Freeze, R. A. and Cherry, J. A.: Groundwater, Prentice-Hall, N.J, 1979.

Gerolymatou, E., Vardoulakis, I., and Hilfer, R.: Modelling infiltration by means of a nonlinear fractional diffusion model, J. Phys. D Appl. Phys., 39, 4104-4110, 2006.

Guerrini, I. A. and Swartzendruber, D.: Soil water diffusivity as explicitly dependent on both time and water content, Soil Sci. Soc. Am. J., 56, 335-340, 1992.

He, J.-H.: Approximate analytical solution for seepage flow with fractional derivatives in porous media, Comput. Method. Appl. Mech., 167, 57-68, 1998.

Levy, M. and Berkowitz, B.: Measurement and analysis of nonFickian dispersion in heterogeneous porous media, J. Contam. Hydrol., 64, 203-226, 2003.

Li, M.-F., Ren, J. R., and Zhu, T.: Series expansion in fractional calculus and fractional differential equations, arXiv: 0910.4819v2 [math-ph], 2009.

Meerschaert, M. M., Benson, D. A., and Baumer, B.: Multidimensional advection and fractional dispersion, Phys. Rev. E, 59, 5026-5028, 1999.

Meerschaert, M. M., Benson, D. A., Scheffler, H. P., and Baumer, B.: Stochastic solution of space-time fractional diffusion equations, Phys. Rev. E, 65, 041103-1-04113-4, 2002. 
Meerschaert, M. M., Mortensen, J., and Wheatcraft, S. W.: Fractional vector calculus for fractional advection-dispersion, Physica A, 367, 167-181, 2006.

Nielsen, D. R., Biggar, J. W., and Davidson, J. M.: Experimental consideration of diffusion analysis in unsaturated flow problem, Soil Sci. Soc. Am. Pro., 26, 107-111, 1962.

Odibat, Z. M. and Shawagfeh, N. T.: Generalized Taylor formula, Appl. Math. Comput., 186, 286-293, 2007.

Pachepsky, Y., Timlin, D., and Rawls, W.: Generalized Richards' equation to simulate water transport in unsaturated soils, J. Hydrol., 272, 3-13, 2003.

Peaudecerf, G. and Sauty, J.-P.: Application of a mathematical model to the characterization of dispersion effects on groundwater quality, Prog. Water Technol., 10, 443-454, 1978.

Podlubny, I.: Fractional Differential Equations, Academic Press, San Diego, 340 pp., 1999.

Rawlins, S. L. and Gardner, W. H.: A test of the validity of the diffusion equation for unsaturated flow of soil water, Soil Sci. Soc. Am. Pro., 27, 507-511, 1963.

Schumer, R., Benson, D. A., Meerschaert, M. M., and Wheatcraft, S. W.: Eulerian derivation for the fractional advection-dispersion equation, J. Contam. Hydrol., 48, 69-88, 2001.

Schumer, R., Meerschaert, M. M., and Baumer, B.: Fractional advection-dispersion equations for modeling transport at the earth surface, J. Geophys. Res., 114, F00A07, doi:10.1029/2008JF001246, 2009.

Sidle, C., Nilson, B., Hansen, M., and Fredericia, J.: Spatially varying hydraulic and solute transport characteristics of a fractured till determined by field tracer tests, Funen, Denmark, Water Resour. Res., 34, 2515-2527, 1998.
Silliman, S. E. and Simpson, E. S.: Laboratory evidence of the scale effect in dispersion of solutes in porous media, Water Resour. Res., 23, 1667-1673, 1987.

Sudicky, E. A., Cherry, J. A., and Frind, E. O.: Migration of contaminants in groundwater at a landfill: a case study. 4. A naturalgradient dispersion test, J. Hydrol., 63, 81-108, 1983.

Sun, H., Meerschaert, M., Zhang, Y., Zhu, J., and Chen, W.: A fractal Richards' equation to capture the non-Boltzmann scaling of water transport in unsaturated media, Adv. Water Resour., 52, 292-295, 2013.

Usero, D.: Fractional Taylor series for Caputo fractional derivatives. Construction of numerical schemes, http://www.fdi.ucm. es/profesor/lvazquez/calcfrac/docs/paper_usero.pdf (last access: February 2017), 2008.

Zhang, Y., Benson, D. A., Meerschaert, M. M., and Labolle, E. M.: Space-fractional advection-dispersion equations with variable parameters: diverse formulas, numerical solutions, and application to the macrodispersion experiment site data, Water Resour. Res., 43, W05439, doi:10.1029/2006WR004912, 2007.

Zhang, Y. and Benson, D. A.: Lagrangian simulation of multidimensional anomalous transport at the MADE site, Geophys. Res. Lett., 35, L07403, doi:10.1029/2008GL033222, 2008.

Zhang, Y., Benson, D. A., and Reeves, D. M.: Time and space nonlocalities underlying fractional-derivative models: distinction and literature review of field applications, Adv. Water Resour., 32, 561-581, 2009. 